\title{
Thermoelectric Generation Based on Spin Seebeck Effect in NiFeCuMo Alloy
}

\author{
B. Guzowski*, R. Gozdur and M. Lakomski \\ Lodz University of Technology, Department of Semiconductor and Optoelectronics Devices, \\ 211/215 Wolczanska Str., 90-924 Lodz, Poland
}

\begin{abstract}
An overview of the achieved Inverse Spin Hall Effect voltage $\left(V_{I S H E}\right)$ is presented to find upper limit of this $V_{I S H E}$. Comprehensive review confirms that the most significant spin systems are based on YIG substrate. The Pt ISHE interfaces are the most popular, however, the best result was reported for the $\operatorname{Ir}_{20} \mathrm{Mn}_{80}$ ISHE interface. Moreover, in this paper the transvers spin Seebeck effect (SSE) is measured in bulk sample of $\mathrm{Ni}_{76.1} \mathrm{Fe}_{15.9} \mathrm{Cu}_{4.3} \mathrm{Mo}_{3.6}$ with $\mathrm{Pt}$ interface. The max. measured value of $V_{I S H E}$ for NiFeCuMo alloy with $\mathrm{Pt}$ is $0.493 \mu \mathrm{V}$ with $\Delta T=21.5 \mathrm{~K}$.

DOI: 10.12693/APhysPolA.133.541
\end{abstract}

PACS/topics: 75.76.+j, 85.80.Fi, 72.15.Jf

\section{Introduction}

Generating of pure spin current in magnetic systems has attracted much attention since it can be controlled and changed much faster and requires less energy than the charge current. Spin Hall effect (SHE), despite of spin pumping and SSE, allows to generate spin current, while the inverse spin Hall effect (ISHE) allows to detect it. The first observation of Spin Seebeck effect (SSE) was made with the use of the ISHE [1].

The SSE has been observed in two configurations either transverse (TSSE) or longitudinal (LSSE), where spin currents were respectively measured perpendicular and parallel to $\nabla T$. The first observation of the TSSE by using a junction system comprising of ferromagnetic film $\mathrm{Ni}_{81} \mathrm{Fe}_{19}$ and $\mathrm{Pt}$ wires was reported in 208 [1] while the LSSE configuration has been presented two years later [2]. Ferrimagnetic insulator Y3Fe5O12 (YIG) and as previously $\mathrm{Pt}$ wires were used in this configuration. The SSE can be disturbed e.g. by the Nernst-Ettingshausen effects [3] or thermal conductive mismatches [4].

Since these first reports a great number of articles related to the observation of SSE in various combinations of substrates and films has been published. In this paper the presence of SSE in bulk sample of $\mathrm{Ni}_{76.1} \mathrm{Fe}_{15.9} \mathrm{Cu}_{4.3} \mathrm{Mo}_{3.6}$ (mumetall) with the Pt interface is proven. Detailed description of the measurement setup and results are given.

\section{Review of materials used as magnetic insulator and conductive films}

The SSE effect was observed in ferromagnetic, ferrimagnetic, paramagnetic [5] and antiferromagnetic materials $[6]$.

\footnotetext{
* corresponding author; e-mail: bartlomiej.guzowski@p.lodz.pl
}

When considering the electrical conductivity of the magnetic substrate, SSE has been observed in metals [1] and semiconductors [7], however, the most appropriate material for a substrate are magnetic insulators since they reduce the Nernst and damping effects. The combination of YIG and Pt is still a referring system to the conducted research with different magnetic insulators and conductive films. In general magnetic insulators can be divided into the following groups [8]:

$$
\text { garnet ferrites: } \quad \mathrm{Y}_{3} \mathrm{Fe}_{5} \mathrm{O}_{12} \quad \text { (YIG), }
$$
$\mathrm{Y}_{3-x} \mathrm{R}_{x} \mathrm{Fe}_{5-y} \mathrm{M}_{y} \mathrm{O}_{12} \quad(\mathrm{R}=\mathrm{Ca}, \mathrm{Nd}, \mathrm{Gd}, \mathrm{Bi} ; \mathrm{M}=\mathrm{Al}$, $\mathrm{Mn}, \mathrm{Ga}, \mathrm{V}, \mathrm{In}, \mathrm{Zr}), \mathrm{Gd}_{3} \mathrm{Fe}_{5} \mathrm{O}_{12}, \mathrm{Gd}_{3} \mathrm{Ga}_{5} \mathrm{O}_{12}$, spinel ferrites: (Mn, Zn) $\mathrm{Fe}_{2} \mathrm{O}_{12}, \mathrm{Fe}_{3} \mathrm{O}_{4}, \mathrm{NiFe}_{2} \mathrm{O}_{4}$, $\mathrm{Co}_{x} \mathrm{Fe}_{3-x} \mathrm{O}_{4} \quad(\mathrm{x}=0.25, \quad 0.75, \quad 1), \quad \mathrm{CoCr}_{2} \mathrm{O}_{4}$, $\mathrm{Ni}_{0.2} \mathrm{Zn}_{0.3} \mathrm{Fe}_{2.5} \mathrm{O}_{4}$,

$$
\text { hexagonal ferrites: } \mathrm{BaFe}_{12} \mathrm{O}_{19} \text {, }
$$

$\mathrm{Ba}_{0.5} \mathrm{Sr}_{1.5} \mathrm{Zn}_{2} \mathrm{Fe}_{12} \mathrm{O}_{22}$, perovskites: $\quad \mathrm{La}_{2} \mathrm{NiMnO}_{6}, \quad \mathrm{La}_{0.67} \mathrm{Sr}_{0.33} \mathrm{MnO}_{3}$,

$\mathrm{DyScO}_{3}$,

others: $\mathrm{Cr}_{2} \mathrm{O}_{3}, \mathrm{MnF}_{2}$.

Likewise, the conductive films can also be grouped as follows [8]:

pure elements: $\mathrm{Pt}, \mathrm{Au}, \mathrm{Ir}, \mathrm{Pd}, \mathrm{Ni}, \mathrm{W}, \mathrm{Ta}, \mathrm{Mo}, \mathrm{Nb}$, $\mathrm{Cr}, \mathrm{Ti}$,

alloys: $\mathrm{NiFe}, \mathrm{FePt}, \mathrm{IrMn}, \mathrm{CoFeMo}$

metallic bilayers: $\mathrm{Pt} / \mathrm{x}(\mathrm{x}=\mathrm{Cu}, \mathrm{Au}, \mathrm{FeCu}, \mathrm{Ti})$, $\mathrm{CoFeB} / \mathrm{Ti}, \mathrm{Co} / \mathrm{Cu}$,

oxides: $\mathrm{IrO}_{2}, \mathrm{SrRuO}_{3}$.

The largest number of articles report on observation of SSE in magnetic insulators YIG [2, 9-21]. Achieved Inverse Spin Hall Effect voltage $\left(V_{I S H E}\right)$ in bilayers are ranging from 0.008 to $20 \mu \mathrm{V}$. The most popular interface in bilayers is $\mathrm{Pt}[1,5,9,10,12,15,21-38]$. The values of $V_{I S H E}$ for bilayers with $\mathrm{Pt}$ are ranging from 0.03 to $12 \mu \mathrm{V}$. In great number of the given references, the SSE were observed in defined $\Delta T$. However, in $[5,11]$ alternative sources of heating using resistive heating layers, light absorption [21,37] or microwave [36] were applied. To make the presented values of $V_{I S H E}$ more comparable only bilayers (except thermopiles in [9] where 
$V_{I S H E}=120 \mu \mathrm{V}$ ) were compared. Some of the presented values were estimated indirectly from charts. Moreover, some of the reached $V_{I S H E}$ values may contain the anomalus Nernst effect (ANE) voltage constitutent.

\section{Measurement setup}

The ferromagnetic/Pt type bilayer was used for investigations of TSSE. The tested sample was made of commercial NiFe type alloy with deposited Pt contacts. Mumetall alloy from Vacuumschmelze was applied for the tests after annealing to improve its static permeability (250000) and reduce coercivity below $0.15 \mathrm{~A} / \mathrm{m}$. EDS analysis indicates composition of $\mathrm{NiFe}$ with dopants of $\mathrm{Cu}$ and Mo (Fig. 1, Table I). The $4 \times 8 \times 0.15[\mathrm{~mm}]$ sample of mumetall was used as ferromagnetic substrate and $\left(1 \times 8 \times 10^{-5}\right)[\mathrm{mm}] \mathrm{Pt}$ contacts were placed on the substrate according to the scheme in Fig. 2.

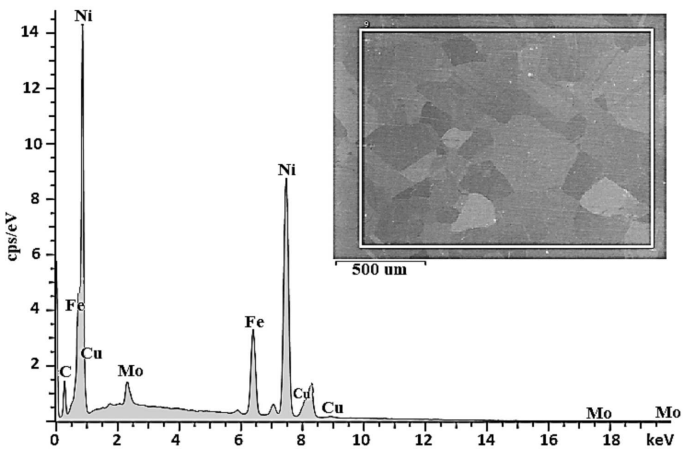

Fig. 1. The EDS spectrum of NiFeCuMo. Inset shows NiFeCuMo structure.

The nominal and the measured

TABLE I NiFeCuMo composition, [wt.\%]

\begin{tabular}{c|c|c|c|c}
\hline \hline Element & $\mathrm{Ni}$ & $\mathrm{Fe}$ & $\mathrm{Cu}$ & $\mathrm{Mo}$ \\
\hline nominal & 76.6 & 14.7 & 4.5 & 3.3 \\
measured & 76.1 & 15.9 & 4.3 & 3.5
\end{tabular}

In Fig. 2a scheme of the setup for measuring the transverse configuration of SSE is shown Temperature gradient $\Delta T=T_{h}-T_{c}$ was generated by thermoelectric cooling module (TEC) placed under the warmer side of the bulk sample. The second side of the bulk sample was placed on the Al block. The generated $\Delta T$ was controlled by 9 Watts TEC module $(15 \times 15)[\mathrm{mm}]$ and measured by two PT100 temperature sensors. Over the bulk sample $\Delta T$ was applied in the $x$ direction perpendicular to the $\mathrm{Pt}$ interface. The presented system was placed between the poles caps of the H-frame electromagnet with digitally adjustable magnetic field. The setup with the sample was placed to render consistent direction of magnetic field strength $H$ in the electromagnet gap with temperature gradient $\Delta T$ The $V_{I S H E}$ was measured between the ends of the $\mathrm{Pt}$ interface placed on a cooler side of bulk sample with the nanovoltmeter Keysight 34420A.

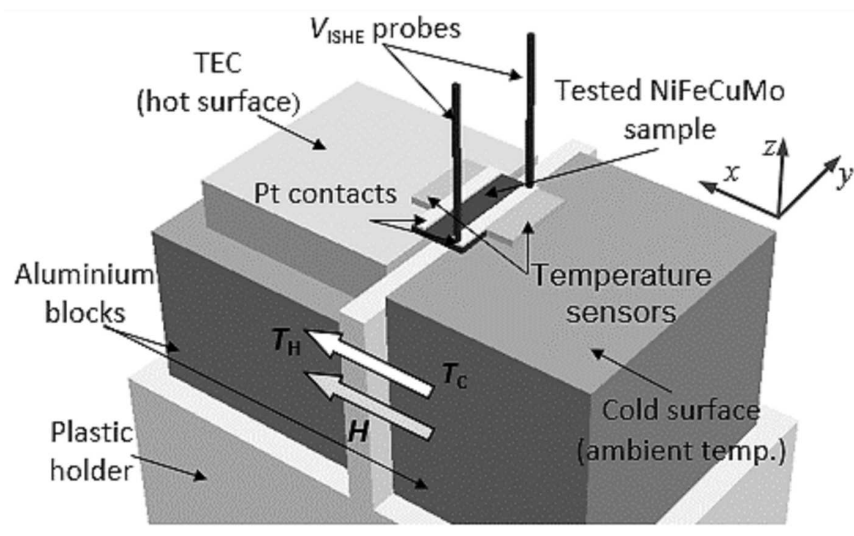

Fig. 2. A scheme of transverse setup for observing SSE.

\section{Results and discussion}

All tests were performed five times. The presented data is the average of the obtained values. In Fig. 3 the $\Delta T$ dependence of $V_{I S H E}$ is presented. For these tests the magnetic field strength $H$ was set to $22.45 \mathrm{kA} / \mathrm{m}$ and temperature of the ambient was $297 \mathrm{~K}$. The influence of seven gradients (from $\Delta T=1 \mathrm{~K}$ to $\Delta T=21.5 \mathrm{~K}$ ) of temperature on the generation of $V_{I S H E}$ was investigated. The measured values of $V_{I S H E}$ increased with increasing the $\Delta T$ and are in the range of 0.031 to $0.319 \mu \mathrm{V}$.

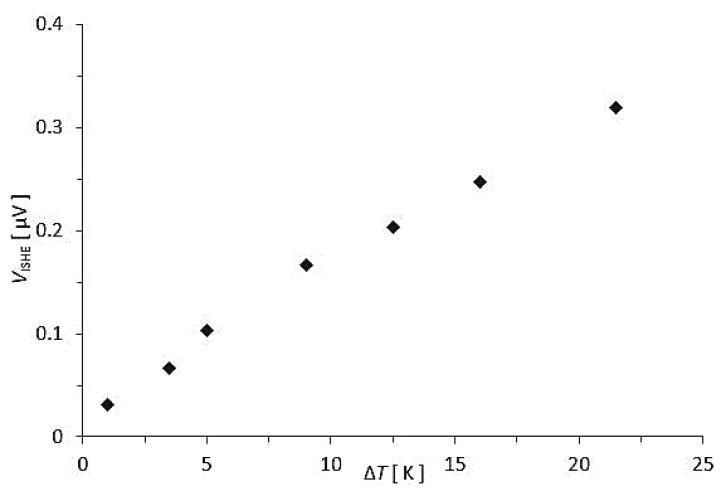

Fig. 3. $\Delta T$ dependence of $V_{I S H E}$ for $H=22.45 \mathrm{kA} / \mathrm{m}$ in $297 \mathrm{~K}$ temperature.

In Fig. 4 the $H$ dependence of $V_{I S H E}$ for three different $\Delta T$ was investigated. For $\Delta T=0 \mathrm{~K}$ the values of $V_{I S H E}$ were stable in the range of $0.038 \div 0.048 \mu \mathrm{V}$ for $7.56<H<50 \mathrm{kA} / \mathrm{m}$. For $\Delta T=12.5 \mathrm{~K}$ by increasing the value of $H$ from 0 to $25 \mathrm{kA} / \mathrm{m}$ the $V_{I S H E}$ raised from 0 to $0.229 \mu \mathrm{V}$. Further increasing of $H$ does not affect the $V_{I S H E}$ and the value of $V_{I S H E}$ fluctuates around $0.225 \mu \mathrm{V}$. The maximal measured value of $V_{I S H E}$ for $\Delta T=12.5 \mathrm{~K}$ was $0.266 \mu \mathrm{V}$.

By increasing the $\Delta T$ to $21.5 \mathrm{~K}$ the highest values of $V_{I S H E}$ were measured The highest $V_{I S H E}=0.493 \mu \mathrm{V}$ was measured for $\Delta T=21.5 \mathrm{~K}$ and $H=49.60 \mathrm{kA} / \mathrm{m}$. 


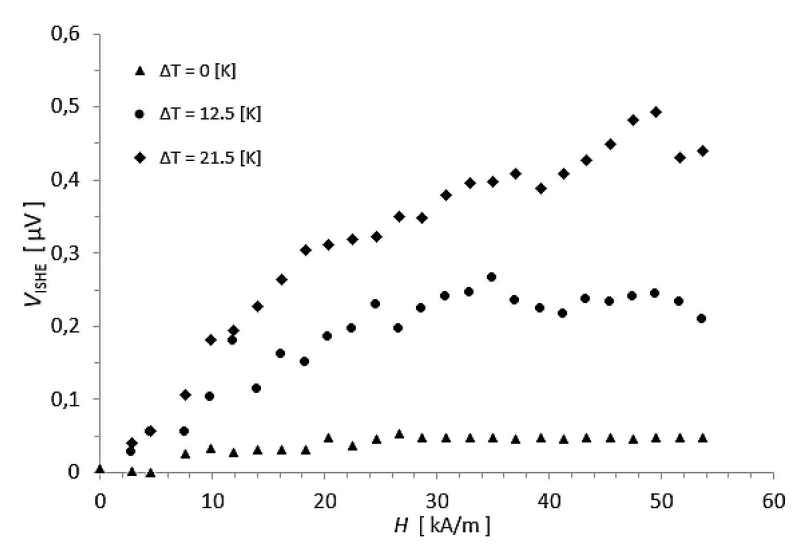

Fig. 4. $H$ dependence of $V_{I S H E}$ for three values of $\Delta T: 0 \mathrm{~K}, 12.5 \mathrm{~K}$ and $21.5 \mathrm{~K}$, measured on Pt interface attached to the lower-temperature side of bulk sample $\mathrm{NiFeCuMo}$ alloy.

\section{Conclusions}

The systems based on YIG substrates are the most popular, however, the structures based on $\mathrm{NiFe} / \mathrm{Pt}$ seem to be more attractive in applications for thermogenerators and sensory technologies. We proved that SSE is also observed in NiFeCuMo alloys with $\mathrm{Pt}$ interface. The maximum measured value of $V_{I S H E}$ for NiFeCuMo alloy with $\mathrm{Pt}$ is $0.493 \mu \mathrm{V}$ with $\Delta T=21.5 \mathrm{~K}$ and $H=49.60 \mathrm{kA} / \mathrm{m}$. By reducing $H$ by half the value of measured $V_{I S H E}$ dropped to $0.319 \mu \mathrm{V}$. Both values of $V_{I S H E}$ are sufficient for sensory applications.

\section{References}

[1] K. Uchida, et al., Nature 455, 778, (2008).

[2] K. Uchida, et al., Appl. Phys. Lett 97, 172505, (2010).

[3] S. R. Boona et al., Energy Environ. Sci. 7, 885 (2014).

[4] S. Y. Huang, et al., Phys. Rev. Lett 107, 216604/1 (2011).

[5] S.M. Wu et al., Phys. Rev. Lett. 114, 186602 (2015).

[6] S.M. Wu et al., Phys. Rev. Lett. 116, 097204 (2016).
[7] C.M. Jaworski, et al., Phys. Rev. Lett 106, 186601 (2011).

[8] K. Uchida, et al., Proc. of IEEE. 104, 1499, (2012).

[9] K. Uchida et al., App. Phys. Exp. 5, 093001 (2012).

[10] T. Kikkawa et al., Phys. Rev. Lett. 110, 067207 (2013).

[11] Y. Xu et al., Appl. Phys. Lett. 105, 242404 (2014).

[12] D. Qu et al., Phys. Rev. B 89, 140407(R), (2014).

[13] D. Qu et al., Phys. Rev. B 92, 020418(R) (2015).

[14] B.F. Miao et al., Phys. Rev. Lett. 111, 066602 (2013).

[15] J. B. S. Mendes et al., Phys. Rev. B 89, 140406(R) (2014).

[16] L.K. Zou et al., Phys. Rev. B 93, 014422 (2016).

[17] A. Azevedo et al., App. Phys. Lett. 104, 052402 (2014).

[18] Z. Qiu et al., Appl. Phys. Express 8, 083001 (2015).

[19] D. Tian et al., App. Phys. Lett. 106, 212407 (2015).

[20] T. Seki et al., Appl. Phys. Lett. 107, 092401 (2015).

[21] M. Schreier et al., Phys. Rev. B 88, 094410 (2013).

[22] S. Geprags et al., Nature Communications 7, 10452, (2016).

[23] S.M. Wu et al., Phys. Rev. Lett. 116, 097204 (2016).

[24] H. Asada et al., Physics Procedia 75, (2015).

[25] P. Li et al., Appl. Phys. Lett. 105, 242412 (2014).

[26] K. Uchida et al., Applied Physics Letters 97, 262501 (2010).

[27] T. Niizeki et al., AIP Advances 5, 053603 (2015).

[28] D. Meier et al., Phys. Rev. B 87, 054421, (2013).

[29] R. Ramos et al., Appl. Phys. Lett. 102, 072413 (2013).

[30] K. Uchida et al., Nature Materials 9, 894-897 (2010).

[31] R. Ramos et al., Phys. Rev. B 92, 220407(R) (2015).

[32] S. Seki et al., Phys. Rev. Lett. 115, 266601 (2015).

[33] R. Takagi et al., APL Mater 4, 032502 (2016).

[34] E. Guo et al., Appl. Phys. Lett. 108, 022403 (2016).

[35] A. Kirihara et al., Scientific Reports 6, 23114 (2016).

[36] Y. Shiomi, E. Saitoh, Phys. Rev. Lett. 113, 266602 (2014).

[37] K.-D. Lee et al., Sci. Rep 5, 10249 (2015).

[38] S. Wu et al., J. App. Phys. 117, 17 C509 (2015). 